\title{
Pour en lire plus : Éducation à l'environnement vers des publics en situation de précarité économique et sociale
}

Étienne van Steenberghe

\section{(2) OpenEdition}

Édition électronique

URL : http://journals.openedition.org/ere/566

DOI : 10.4000/ere.566

ISSN : 2561-2271

Éditeur

Centr'ERE

Référence électronique

Étienne van Steenberghe, «Pour en lire plus : Éducation à l'environnement vers des publics en situation de précarité économique et sociale», Éducation relative à l'environnement [En ligne], Volume 12 | 2015, mis en ligne le 20 mai 2015, consulté le 24 septembre 2020. URL : http:// journals.openedition.org/ere/566; DOI : https://doi.org/10.4000/ere.566 
Pour en lire plus : Éducation à l'environnement vers des publics en situation de précarité économique et sociale

\author{
Étienne van Steenberghe
}

\title{
RÉFÉRENCE
}

Hortalan, M. (Dir.) (2012). Éducation à l'environnement vers des publics en situation de précarité économique et sociale. Collection « Les livrets de l'Ifrée », no 4, décembre 2012, $129 \mathrm{p}$. 


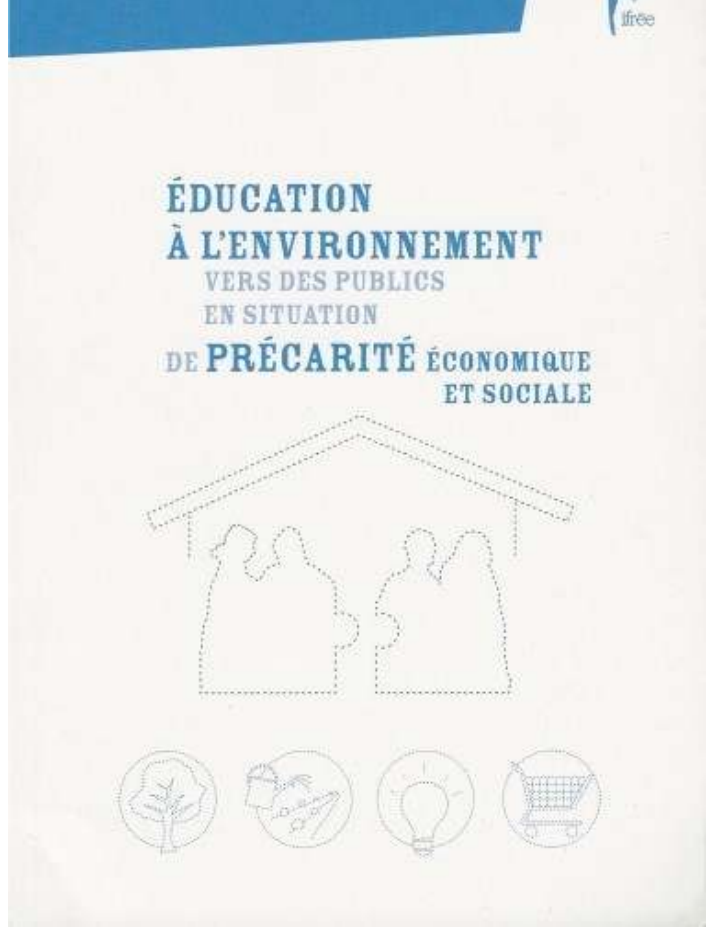

Enracinées dans le réel, les pratiques de l'éducation à l'environnement se construisent et se développent en tenant compte des savoirs locaux. Mais quand il s'agit de mettre en place un projet auprès de publics en situation de grande précarité économique et sociale, les éducateurs sont souvent démunis. Dans beaucoup de cas, ils doivent interagir avec des personnes qui se retrouvent en rupture avec la société, avec le système éducatif, avec les autres. Pour cette raison, ce public est souvent oublié dans les actions de terrain. C'est pour remédier à cette situation que l'Institut de formation et de recherche en éducation à l'environnement (Ifrée) a décidé d'y consacrer un numéro de sa collection des « livrets». Cette publication se subdivise en deux grandes sections : «Des pratiques actuelles » et «Comment s'y mettre? »

2 Comme pour les autres livrets, la première partie s'appuie entièrement sur des expériences vécues par des praticiens. On y recueille un ensemble d'activités centrées sur quatre thématiques classiques liées à l'éducation à l'environnement : la nature, les jardins collectifs et l'alimentation, l'eau et l'énergie, la consommation et les déchets. À titre d'exemple, mentionnons l'implantation de jardins partagés, des ateliers de cuisine, des activités de découvertes de la faune et de la flore, une exposition présentant des astuces pour économiser l'eau ou l'énergie, etc. Les actions éducatives présentées dans cette section du livret sont toutes issues de rencontres entre différents acteurs sociaux et partenaires privilégiés. Les populations défavorisées ciblées s'impliquent ensuite à travers des ateliers de sensibilisation, des rencontres et des démarches d'accompagnement; cette dynamique de groupe peut parfois orienter autrement les activités prévues au départ. Nous pouvons cependant regretter que la majorité de ces projets ne soient pas envisagés et co-construits directement par les personnes concernées en s'inscrivant dans une démarche préalable qui prendrait en compte les besoins réels, tels qu'ils sont exprimés par la collectivité. Il nous semble en effet important d'appréhender dans un premier temps, les raisons qui conduisent les personnes à agir. Mais nous sommes aussi conscients de la difficulté d'amener un tel 
public à mettre en place et à réaliser de tels projets qui seraient "leurs", les éducateurs à l'environnement devenant alors des accompagnateurs. En outre, une telle démarche pourrait mener à prendre conscience que les besoins et les attentes des personnes défavorisées n'autorisent plus de s'en tenir à une simple intervention d'éducation à l'environnement.

3 Également, dans cette première partie du livret, en s'appuyant sur les exemples concrets, certaines pistes de dispositifs participatifs sont présentées pour tenter de répondre aux interrogations des éducateurs en environnement quant à l'adaptation possible de leurs actions avec des publics en situation de précarité économique et sociale. On en conclut entre autres que les animateurs doivent faire preuve d'une grande adaptabilité, d'empathie et surtout, se centrer prioritairement sur ce qui a une réelle signification pour le public en question. Les acteurs sont ainsi invités à sortir d'un « entre soi » socioculturel (« le champ thématique de l'environnement a toujours été porté par des représentants de la classe moyenne », p. 71) et réinterroger leurs repères et les paradigmes qui guident leurs actions. Une posture adaptée est donc celle de l'écoute et de l'ouverture afin d'entrer en relation et de tenter de comprendre le public cible des interventions. Il s'agit aussi de sortir de nos préjugés et d'envisager ces personnes comme des participants, comme des experts de leur situation et des acteurs de changement. On le voit : le contact humain donc essentiel quand on travaille avec ce type de public. Notons enfin que de nombreuses contraintes organisationnelles doivent être prises en compte: elles sont mentionnées au fil des divers projets : contraintes temporelles, contraintes méthodologiques pour le développement d'outils de vulgarisation et contraintes communicationnelles.

La seconde partie de ce livret se centre sur l'amont, soit la construction du projet d'éducation. C'est le «comment s'y mettre?» Après avoir abordé les questions de précarité et ses répercussions sur les personnes, et 'approfondi l'intérêt de mener des actions d'éducation à l'environnement avec celles-ci, il s'agit de se questionner sur la préparation du projet et sa conduite pédagogique, sur le partenariat avec le secteur social, la mobilisation du public, la mixité sociale dans les actions, et l'adaptation du dispositif pédagogique et des pratiques éducatives à mettre en oeuvre. Tous ces points sont largement détaillés. Nous nous limiterons ici à deux commentaires: la construction de partenariat et la mobilisation des participants. Premièrement, un projet d'éducation à l'environnement avec ce public demande une multicompétence qui s'articule autour d'un axe environnemental et social, voire de santé publique. Or le monde du travail social et de l'éducation à l'environnement, même s'ils partagent des valeurs communes, n'ont pas l'habitude de travailler ensemble. Par ailleurs, si l'inscription d'un projet d'éducation à l'environnement dans un partenariat avec une institution peut être une assurance de sérieux pour les participants, il peut aussi être un obstacle, car souvent, ces personnes ont développé une certaine méfiance vis-à-vis de ces institutions. Deuxièmement, dans les situations présentées dans ce livret, ce sont des professionnels qui vont être le point de départ des actions éducatives. On peut dès lors relever un ensemble de difficultés dans la participation citoyenne d'un public qui montre le plus souvent une grande défiance. D'ailleurs, plusieurs exemples présentés dans ce document posent la question de la participation - dans le sens de "prendre sa part à » - des personnes défavorisées si l'on veut que le projet entrepris ait du sens pour celle-ci. Construire le projet à partir de ce public et avec celui-ci, perçu comme acteur, 
serait un moyen de reconnaitre et de valoriser les personnes en situation de précarité économique et sociale.

En conclusion, l'ensemble des projets présentés dans ce livret répond à la visée d'enrichir nos pratiques et d'améliorer les situations vécues individuellement et collectivement par les personnes défavorisées. Ce document pédagogique est de nature à susciter une démarche réflexive. Il devrait permettre à de nouveaux acteurs locaux de trouver les moyens de concrétiser leur engagement auprès d'un public fragilisé socialement et économiquement dans le but de renforcer le pouvoir d'agir de ces personnes sur leur environnement quotidien de proximité.

\section{AUTEURS}

\section{ÉTIENNE VAN STEENBERGHE}

Chercheur associé, Centre de recherche en éducation et formation relatives à l'environnement et à l'écocitoyenneté, Université du Québec à Montréal 